\title{
Diffusion Coefficients at Infinite Dilution in Water and in $N$-Methylpyrrolidone
}

\author{
Marcel J. M. te Riele, Erwin D. Snijder, and Wim P. M. van Swaaij* \\ Department of Chemical Engineering, Twente University of Technology, \\ P.O. Box 217, 7500 AE Enschede, The Netherlands
}

The Taylor dispersion method has been used to determine diffusion coefficients of $N$-methylpyrrolidone, 2-methyl-2,4-pentanediol, glycerol, 1,3-butanediol, and 1,2,6-hexanetriol at infinite dilution in water and of water, glycerol, and 2-methyl-2,4-pentanediol at infinite dilution in $N$-methylpyrrolidone. The temperature has been varied between 298 and $348 \mathrm{~K}$.

\section{Introduction}

Membrane formation and polymer spinning processes can be described as ternary or higher phase systems (polymer, solvent, nonsolvent, additives). Important for the description of these processes are diffusion coefficients of the different components (1) in both the solvent $(N$ methylpyrrolidone) and nonsolvent (water). In the present work the diffusion coefficients of some relevant solvent, nonsolvent, or additive systems have been determined at infinite dilution.

Various techniques are available for the determination of diffusion coefficients. In the present work the TaylorAris dispersion method has been used to measure the diffusion coefficients. The required theory was originally derived by Taylor and extended by Aris $(2-4)$. A brief treatment of the theory is presented in the next section, more details are provided by, e.g., Alizadeh et al. (5), Baldauf and Knapp (6), and Snijder et al. (7).

\section{Theory}

When a pulse of tracer is injected into a solvent flowing laminary through a tube, the combination of axial convection and radial diffusion will cause a spreading of the solute $(2,3)$. Taylor derived that under certain conditions the concentration profile can be approximated as

$$
C_{\mathrm{m}}=\frac{N_{\text {inj }}}{2 \pi R^{2}(\pi K t)^{1 / 2}} \exp \left[-\frac{(x-u t)^{2}}{4 K t}\right]
$$

where $C_{\mathrm{m}}$ is the averaged radial concentration, $u$ the average flow velocity, $R$ the tube radius, $x$ the axial coordinate, $t$ time, and $N_{i n j}$ the injected amount of solute. Taylor has given a relation for the axial dispersion coefficient $K$, assuming that axial molecular diffusion can be neglected as compared to axial convection:

$$
K=u^{2} R^{2} / 48 D
$$

Aris (4) has demonstrated that a more general equation for the dispersion coefficient $K$ can be obtained when molecular diffusion in the axial direction is taken into account as well:

$$
K=u^{2} R^{2} / 48 D+D
$$

In a practical setup the injection of a solute pulse will be of finite length. However, as long as the pulse length is less than $1 \%$ of the capillary length, the pulse can be regarded as a $\delta$-Dirac injection $(6,8)$. The tube length

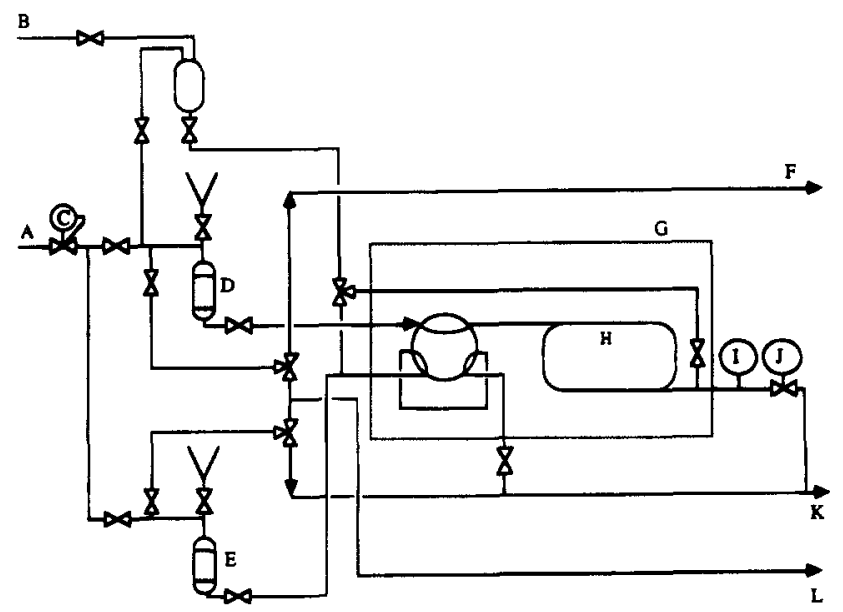

Figure 1. Apparatus: (A) helium, (B) calibration sample, (C) pressure controller, (D) solvent, (E) solute, (F) purge, (G) thermostated water bath, $(\mathrm{H})$ curved coil, (I) detector, $(\mathrm{J})$ mass flow controller, $(\mathrm{K})$ purge, $(\mathrm{L})$ vacuum pump.

required for obtaining accurate results is generally several meters. Therefore, a curved tube has been used. In that case centrifugal forces $(9,10)$ may cause the development of secondary flow. Janssen has demonstrated that the influence of the centrifugal forces is related to the $(D e)^{2}(S c)$ number. Alizadeh et al. (5) and Snijder et al. (7) showed that above a certain critical $(D e)^{2}(S c)$ number the calculated diffusion coefficient increases with increasing $(D e)^{2}(S c)$. Below this value the influence of secondary flow can be neglected. The $(D e)^{2}(S c)$ number can be written as

$$
(D e)^{2}(S c)=4 R^{2} u^{2} \varrho / \eta R_{c} D
$$

In the present work the critical $(D e)^{2}(S c)$ number has been obtained experimentally by varying the average velocity $u$ at $298 \mathrm{~K}$. Above the critical velocity, the calculated diffusion coefficient started to increase. Results similar to those reported earlier (7) were obtained; the critical $(D e)^{2}(S c)$ appeared to be about 100 . The velocity applied for all experiments was then chosen well below the critical average velocity $u_{\text {crit }}$.

\section{Experimental Section}

The experimental arrangement is shown schematically in Figure 1. The average concentration at the end of the tube is measured by means of a differential refractive index detector (Varian, RI-4). Two glass vessels, containing the 
Table 1. Dimensions of the Apparatus

$\begin{array}{llll}\text { capillary length } L_{\mathrm{c}} & 15.085 \mathrm{~m} & \text { capillary volume } V_{\mathrm{c}} & 1.47 \times 10^{-5} \mathrm{~m}^{3} \\ \text { radius of the tube } R & 5.565 \times 10^{-4} \mathrm{~m} & \text { injected volume } V_{\text {inj }} & 8.7 \times 10^{-8} \mathrm{~m}^{3} \\ \text { curvature of the coil } R_{\mathrm{c}} & 0.1 \mathrm{~m} & \text { detector volume } V_{\text {det }} & 8 \times 10^{-9} \mathrm{~m}^{3}\end{array}$

Table 2. Average Diffusion Coefficients at Infinite Dilution in Water

\begin{tabular}{cccccc}
\hline & \multicolumn{3}{c}{$10^{9} \mathrm{D} /\left(\mathrm{m}^{2} \mathrm{~s}^{-1}\right)$} \\
\cline { 2 - 6 }$T / \mathrm{K}$ & $N$-methylpyrrolidone & 2-methyl-2,4-pentanediol & glycerol & 1,3-butanediol & 1,2,6-hexanetriol \\
\hline 298.0 & 0.946 & 0.746 & 0.971 & 0.863 & 0.755 \\
307.7 & & & & & 0.964 \\
307.9 & & & & & \\
308.0 & 1.208 & 0.963 & 1.234 & & \\
318.0 & 1.504 & 1.215 & 1.539 & 1.394 & 1.120 \\
333.0 & 1.996 & 1.639 & 2.035 & 1.870 & 1.614 \\
348.0 & 2.567 & 2.138 & 2.617 & 2.417 & 2.078 \\
std dev & $3.7 \times 10^{-3}$ & $3.3 \times 10^{-3}$ & $2.6 \times 10^{-3}$ & $2.0 \times 10^{-3}$ & $2.3 \times 10^{-3}$
\end{tabular}

Table 3. Average Diffusion Coefficients at Infinite Dilution in $\boldsymbol{N}$-Methylpyrrolidone

\begin{tabular}{lcll}
\hline & \multicolumn{3}{c}{$10^{9} \mathrm{D} /\left(\mathrm{m}^{2} \cdot \mathrm{s}^{-1}\right)$} \\
\cline { 2 - 4 }$T / \mathrm{K}$ & 2-methyl-2,4-pentanediol & glycerol & water \\
\hline 298.1 & 0.597 & 0.488 & 1.003 \\
308.0 & 0.729 & 0.592 & 1.164 \\
318.0 & 0.865 & 0.715 & 1.514 \\
333.0 & 1.086 & 0.915 & 1.987 \\
348.0 & 1.346 & 1.154 & 2.546 \\
std dev & $2.3 \times 10^{-3}$ & $4.6 \times 10^{-3}$ & $1.2 \times 10^{-2}$
\end{tabular}

solvent and the solute are kept under constant helium pressure ( 5 bar) by means of a precision pressure reduce valve. The flow through the capillary tube is regulated with a mass flow controller (Rosemount Flowmega 5881) located after the detector. This configuration assures a completely constant and pulsation-free flow throughout the experiment. The solute pulse is introduced by switching a helium actuated injection valve (switching time: $0.1 \mathrm{~s}$ ). The capillary tube is elliptically coiled and placed in a water bath (the temperature is constant within $0.1 \mathrm{~K}$ ). Table 1 lists the dimensions of the apparatus.

The procedure for the measurement of diffusion coefficients is described in detail by Snijder et al. (7). Therefore, in the present work, it will be addressed only briefly. After injection of a solute pulse in the solvent flowing slowly through the capillary tube, the response at the end of the tube is recorded and stored by a computer. Next, the drift in the base line of the refractive index detector is subtracted from the original data points, after which the average velocity $u$, the injected amount of solute $N_{i n j}$, and the dispersion coefficient $K$ are obtained using eq 1. Equation 3 yields the diffusion coefficient $D$. The calculated (eq 1 ) and measured concentration curves coincide very well. This procedure for the determination of a diffusion coefficient for the mixtures studied in the present work appeared to be very fast with a measuring time of $2-3 \mathrm{~h}$ for each data point. The experiment has been completely automated and is computer controlled, making it possible to carry out a series of experiments by varying the temperature according to a programmed schedule without any interruption.

\section{Results}

Tables 2 and 3 give the diffusion coefficients for the components at infinite dilution in water and $N$-methylpyrrolidone at different temperatures. The values represent averaged values of $3-4$ measurements; the averaged standard deviation for each system is included in the tables. The temperature dependencies were expressed as an Arrhenius type of equation, which is graphically illustrated in Figure 2 for the systems with $N$-methylpyrrolidone as solvent. This figure also demonstrates that

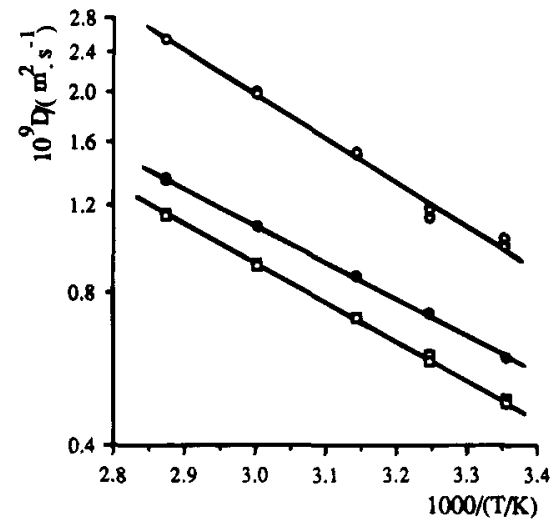

Figure 2. Temperature dependency of the diffusivity in $N$ methylpyrrolidone at infinite dilution: (open circles) water, (closed circles) 2-methyl-2,4-pentanediol, (squares) glycerol.

Table 4. Constants for the Arrhenius Equation (5)

\begin{tabular}{|c|c|c|c|c|}
\hline & \multicolumn{2}{|l|}{ in water } & \multicolumn{2}{|c|}{$\begin{array}{l}\text { in } N \text {-methyl- } \\
\text { pyrrolidone }\end{array}$} \\
\hline & $A$ & $B$ & $A$ & $B$ \\
\hline$N$-methylpyrrolidone & $9.813 \times 10^{-7}$ & 2066 & & \\
\hline 2-methyl-2,4-pentanediol & $11.367 \times 10^{-7}$ & 2180 & $1.660 \times 10^{-7}$ & 1674 \\
\hline glycerol & $9.637 \times 10^{-7}$ & 2052 & $1.949 \times 10^{-7}$ & 1785 \\
\hline 1,3-butanediol & $11.266 \times 10^{-7}$ & 2133 & & \\
\hline 1,2,6-hexanetriol & $8.442 \times 10^{-7}$ & 2087 & & \\
\hline water & & & $7.536 \times 10^{-7}$ & 1980 \\
\hline
\end{tabular}

the spread in the results is very small. The following general relation for the diffusion coefficients as a function of temperature for the various systems has been used

$$
D /\left(\mathrm{m}^{2} \cdot \mathrm{s}^{-1}\right)=A \exp (-B /(T / \mathrm{K}))
$$

with $A$ and $B$ given in Table 4 .

\section{Conclusions}

The diffusion coefficients of several components applied for membrane spinning and preparation processes have been measured at infinite dilution at various temperatures using the Taylor-Aris dispersion method.

\section{Acknowledgment}

We acknowledge $\mathrm{K}$. van Bree, S. Kuipers, W. Leppink, and A. H. Pleiter for their technical support.

\section{Literature Cited}

(1) Boom, R. M. Ph.D. Dissertation, University of Twente, Enschede, The Netherlands, 1992.

(2) Taylor, F. R. S. Proc. R. Soc. London 1953, A219, 186-203.

(3) Taylor, F. R. S, Proc. R. Soc. London 1954, A225, 473-477.

(4) Aris, R. Proc. R. Soc. London 1956, A235, 67-77. 
36 Journal of Chemical and Engineering Data, Vol. 40, No. 1, 1995

(5) Alizadeh, A.; Nieto de Castro, C. A.; Wakeham, W. A. Int. J. Thermophys. 1980, 1, 243-284.

(6) Baldauf, W.; Knapp, H. Chem. Eng. Sci. 1983, 38, 1031-1037.

(7) Snijder, E. D.; te Riele, M. J. M.; Versteeg, G. F,; van Swaaij, W. P. M. J. Chem. Eng. Data 1993, 38, 475-480.

(8) Baldauf, W.; Knapp, H. Ber. Bunsen-Ges. Phys. Chem. 1983, 87 $304-309$.

(9) Nunge, R. J.; Lin, T.-S.; Gill, W. N. J. Fluid Mech. 1972, 51, 363383 .

(10) Janssen, L. A. M. Chem. Eng. Sci. 1976, 31, 215-218.

Received for review August 10, 1993. Revised August 11, 1994. Accepted October $12,1994 \&$ These investigations were supported by the Foundation for Chemical Research in the Netherlands (SON) and by DSM.

JE930156X

${ }^{\otimes}$ Abstract published in Advance ACS Abstracts, December 1, 1994. 